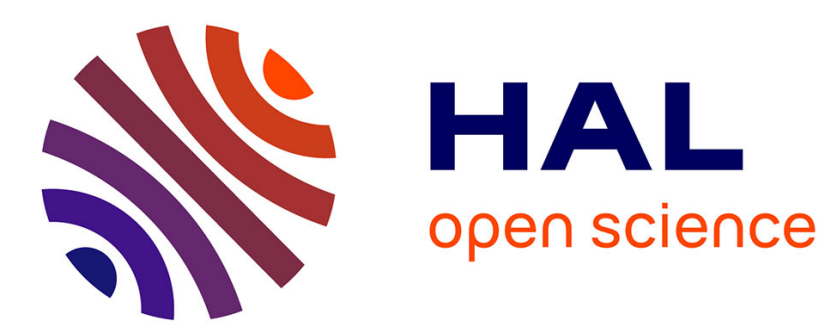

\title{
Estimations du noyau de Green, propriété de valeur moyenne et géométrie des boules hyperboliques
}

\author{
Cyrille Domenichino, Philippe Jaming
}

\section{To cite this version:}

Cyrille Domenichino, Philippe Jaming. Estimations du noyau de Green, propriété de valeur moyenne et géométrie des boules hyperboliques. Comptes rendus de l'Académie des sciences. Série I, Mathématique, 2001, 332, No 12 pp1053-1058. hal-00007480

\section{HAL Id: hal-00007480 \\ https://hal.science/hal-00007480}

Submitted on 12 Jul 2005

HAL is a multi-disciplinary open access archive for the deposit and dissemination of scientific research documents, whether they are published or not. The documents may come from teaching and research institutions in France or abroad, or from public or private research centers.
L'archive ouverte pluridisciplinaire HAL, est destinée au dépôt et à la diffusion de documents scientifiques de niveau recherche, publiés ou non, émanant des établissements d'enseignement et de recherche français ou étrangers, des laboratoires publics ou privés. 


\title{
Estimations du noyau de Green, propriété de valeur moyenne et géométrie des boules hyperboliques
}

\author{
Cyrille DOMENICHINO ${ }^{\text {a }}$, Philippe JAMING ${ }^{\text {b }}$
}

a LAPT (UMR 6632), Université de Provence, 39, rue Joliot-Curie, 13453 Marseille cedex 13, France

b Mapmo (UMR 6628), faculté des sciences, Université d'Orléans, B.P. 6759, 45067 Orléans cedex 2, France Courriel : Cyrille.Domenichino@cmi.univ-mrs.fr ; jaming@labomath.univ-orleans.fr

(Reçu le 6 avril 2001, accepté le 23 avril 2001)

Résumé. Dans cette note, nous obtenons des estimés du noyau de Green dans les boules hyperboliques réelles, complexes et quaternioniques. Celles-ci nous permettent ensuite de montrer que, dans ces boules, les seuls domaines de classe $\mathrm{e}^{1+\alpha}, \alpha>0$ pour lesquels l'égalité de la moyenne surfacique est vraie pour toutes les fonctions harmoniques sont les boules géodésiques. (C) 2001 Académie des sciences/Éditions scientifiques et médicales Elsevier SAS

Green kernel estimates, mean value properties and geometry of classical rank one balls

Abstract. In this Note, we obtain estimates of the Green kernel of real, complex and quaternionic hyperbolic balls. We then apply these to show that in such balls the only domains of class $\mathrm{e}^{1+\alpha}, \alpha>0$ for which the spherical mean value identity holds for every harmonic function are the geodesic balls. (C) 2001 Académie des sciences/Éditions scientifiques et médicales Elsevier SAS

\section{Abridged English version}

In this Note, we denote by $\mathbb{F}=\mathbb{R}, \mathbb{C}$ or $\mathbb{H}$ and $n \geqslant 2$ an integer $(n \geqslant 3$ if $\mathbb{F}=\mathbb{R})$. Let $\mathbb{B}_{n}$ be the Euclidean unit ball of $\mathbb{F}^{n}$. Define $G$ as $\mathbb{F}=\mathbb{R}: G=S O_{0}(n, 1), \mathbb{F}=\mathbb{C}: G=S U(n, 1), \mathbb{F}=\mathbb{H}: G=S p(n, 1)$; and let $G=K A N$ be its Iwasawa decomposition. It is well known that $\mathbb{B}_{n}$ can be identified with $G / K$, in particular $G$ acts on $\mathbb{B}_{n}$ and $\mathbb{B}_{n}$ can be endowed with a $G$-invariant metric. We will refer to this metric as the hyperbolic metric on $\mathbb{B}_{n}$. Further, let $d=\operatorname{dim}_{\mathbb{R}} \mathbb{F}$ and define $m_{1}=d(n-1)$ and $m_{2}=d-1$ the multiplicities of the roots of $G$.

If $z \in \mathbb{B}_{n}$, there exists $g \in G$ such that $g . z=0$ and $g .0=z$. For $\zeta \in \mathbb{B}_{n}$ we will then write $\varphi_{z}(\zeta)=g . \zeta$ so that $\varphi_{z}(\zeta)=\frac{z-P_{z} \zeta-\sqrt{1-\|z\|^{2}} Q_{z} \zeta}{1-\langle\zeta, z\rangle}$ with $P_{z}(\zeta)=\frac{\langle\zeta, z\rangle}{\langle z, z\rangle} z$ and $Q_{z}(\zeta)=\zeta-P_{z}(\zeta)$.

Note présentée par Jean-Pierre KAHANE

S0764-4442(01)01989-9/FLA

(c) 2001 Académie des sciences/Éditions scientifiques et médicales Elsevier SAS. Tous droits réservés. 


\section{Domenichino, P. Jaming}

Denote by $D_{\mathbb{F}}$ the Laplace operator on $\mathbb{B}_{n}$ that is invariant under $G$, by $d \mu$ the $G$-invariant measure on $\mathbb{B}_{n}$. Let $\Omega$ be a relatively compact domain in $\mathbb{B}_{n}$ of class $\mathcal{C}^{1+\alpha}$ such that $\partial \Omega=\partial \bar{\Omega}$. Denote by $d \sigma_{g}$ the contraction of $d \mu$ with regard to the outward normal vector to $\partial \Omega$ with respect to the hyperbolic metric.

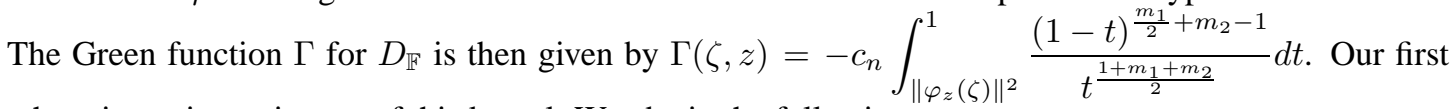
aim here is to give estimates of this kernel. We obtain the following:

Proposition 2.2. For every compact set $K$ of $\mathbb{B}_{n}$, there exists a constant $C_{K}$ such that, for all $z \in K$, $\zeta \in \partial \Omega$ and for $i=1, \ldots, n$

and

$$
\left|\frac{\partial \Gamma}{\partial z_{i}}(z, \zeta)+\frac{\partial \Gamma}{\partial \zeta_{i}}(z, \zeta)\right| \leqslant \frac{C_{K}}{\|z-\zeta\|^{m_{1}+m_{2}-1}}=\frac{C_{K}}{\|z-\zeta\|^{n d-2}}
$$

$$
\left|\frac{\partial \Gamma}{\partial z_{i}}(z, \zeta)\right| \leqslant \frac{C_{K}}{\|z-\zeta\|^{m_{1}+m_{2}}}=\frac{C_{K}}{\|z-\zeta\|^{n d-1}}
$$

From this, we deduce the behaviour of the single-layer potential of $\partial \Omega, u_{\partial \Omega}(z)=\int_{\partial \Omega} \Gamma(\zeta, z) d \sigma_{g}(\zeta)$.

We show that $u_{\partial \Omega}$ is continuous on $\mathbb{B}_{n}$ and :

Proposition 2.3. For every $z_{0} \in \partial \Omega, \frac{\partial u_{\partial \Omega}}{\partial \eta^{+}}\left(z_{0}\right)-\frac{\partial u_{\partial \Omega}}{\partial \eta^{-}}\left(z_{0}\right)=1$, where $\eta$ is the outward normal vector to $\bar{\Omega}$ with respect to the Bergman metric and + (resp. - ) indicates the limit from the exterior (resp. interior).

Sketch of proof. Define the double-layer potential $v_{\partial \Omega}$ of $\partial \Omega$ by $v_{\partial \Omega}(z)=\int_{\partial \Omega}\left\langle\operatorname{grad}_{\zeta} \Gamma(\zeta, z), \eta_{\zeta}\right\rangle d \sigma_{g}(\zeta)$ and we show that $v_{\partial \Omega}(z)=0$ if $z \in \Omega^{+}$, the exterior of $\Omega$ and $v_{\partial \Omega}(z)=1$ if $z \in \Omega$.

Then, for $z_{0} \in \partial \Omega, z=z_{0}+t \eta_{z_{0}}, z^{\prime}=z_{0}-\tau \eta_{z_{0}}, t, \tau>0$

$$
v_{\partial \Omega}(z)-v_{\partial \Omega}\left(z^{\prime}\right)=\int_{\partial \Omega}\left(\left\langle\operatorname{grad}_{z} \Gamma(\zeta, z), \eta_{z_{0}}\right\rangle-\left\langle\operatorname{grad}_{z^{\prime}} \Gamma\left(z^{\prime}, \zeta\right), \eta_{z_{0}}\right\rangle\right) d \sigma_{g}(\zeta) .
$$

By suitably cutting this integral and using the estimates (2.3)-(2.4) as well as the regularity of the boundary, we see that $v_{\partial \Omega}(z)-v_{\partial \Omega}\left(z^{\prime}\right) \rightarrow 1$ as $t, \tau \rightarrow 0$.

Next, we will say that a function $f$ on $\mathbb{B}_{n}$ is $D_{\mathbb{F}}$-harmonic if $D_{\mathbb{F}} f=0$. It is then well known that if $B_{r}$ is an Euclidean ball centered in 0 of radius $r, S_{r}=\partial B_{r}$ and $z \in \mathbb{B}_{n}$, then

$$
f(z)=\frac{1}{\sigma\left(\varphi_{z}\left(S_{r}\right)\right)} \int_{\varphi_{z}\left(S_{r}\right)} f(\zeta) d \sigma_{g}(\zeta)
$$

The estimates above will allow us to prove the following converse of the mean value equality:

Theorem 3.1. Let $\Omega$ be a relatively compact domain in $\mathbb{B}_{n}$, of class $\mathcal{C}^{1+\alpha}, \alpha>0$ such that $\partial \Omega=$ $\partial \bar{\Omega}$. Assume $z \in \Omega$ is such that $f(z)=\frac{1}{\sigma(\partial \Omega)} \int_{\partial \Omega} f(\zeta) d \sigma_{g}(\zeta)$ for every $D_{\mathbb{F}}$-harmonic function in a neighbourhood of $\bar{\Omega}$. Then $\Omega$ is a hyperbolic ball centered at $z$, that is $\Omega=\varphi_{z}\left(B_{r}\right)$ for some $r, 0<r<1$. Proof. The proof is adapted from that of Shahgholian [2] in the Euclidean case and follows closely the complex case from [1]. It is enough to prove the theorem for $a=0$. We will apply $(0.1)$ to the functions $f=\Gamma(z,$.$) with z \notin \bar{\Omega}$. As in [2], we will consider the Euclidean balls centered at $0, B_{1}, B_{2}$, respectively the smallest ball containing $\Omega$ and the biggest ball contained in $\Omega$. We will show that $\partial B_{1}=\partial B_{2}=\partial \Omega$.

By continuity of $u_{\partial \Omega}$ and compacity of $\partial \Omega, u_{\partial \Omega}$ has its minimum and its maximum on $\partial \Omega$ in points $z_{1}$ and $z_{2}$. The hypothesis of the theorem shows that $u_{\partial \Omega}\left(z_{1}\right) \geqslant u_{\partial \Omega}\left(z_{2}\right)$ if and only if $\Gamma\left(z_{1}, 0\right) \geqslant \Gamma\left(z_{2}, 0\right)$ which is equivalent to $\left\|z_{1}\right\| \geqslant\left\|z_{2}\right\|$. In particular $z_{1} \in \partial \Omega \cap B_{1}$ and $z_{2} \in \partial \Omega \cap B_{2}$. Let us now show that $\left\|z_{1}\right\| \leqslant\left\|z_{2}\right\|$.

As $u_{\partial \Omega}$ is $D_{\mathbb{F}}$-harmonic on $\Omega$ and continuous on $\bar{\Omega}, u_{\partial \Omega}$ satisfies the maximum principle. Therefore the supremum of $u$ on $\bar{\Omega}$ is is atteint on $\partial \Omega$ thus in $z_{1}$. Similarly, the infimum of $u$ on $\bar{\Omega}$ is attained in 


\section{Estimations du Noyau de Green sur les boules hyperboliques}

$z_{2}$. It follows that $\frac{\partial u_{\partial \Omega}}{\partial \eta^{-}}\left(z_{1}\right) \geqslant 0$ and $\frac{\partial u_{\partial \Omega}}{\partial \eta^{-}}\left(z_{2}\right) \leqslant 0$. Next, as for Lemma 2 of [1], one may check that $\frac{\partial u_{\partial \Omega}}{\partial \eta^{+}}\left(z_{i}\right)=\left(1-\left\|z_{i}\right\|^{2}\right) \frac{\partial u_{\partial \Omega}}{\partial\|z\|}\left(z_{i}\right)=2 c_{n} \frac{\left(1-\left\|z_{i}\right\|\right)^{\frac{m_{1}}{2}+m_{2}}}{\left\|z_{i}\right\|^{m_{1}+m_{2}}}$. Thus, from Proposition 2.3, one then gets :

$$
\begin{aligned}
& 1=\frac{\partial u_{\partial \Omega}}{\partial \eta^{+}}\left(z_{1}\right)-\frac{\partial u_{\partial \Omega}}{\partial \eta^{-}}\left(z_{1}\right) \leqslant \frac{\partial u_{\partial \Omega}}{\partial \eta^{+}}\left(z_{1}\right)=2 c_{n} \frac{\left(1-\left\|z_{1}\right\|\right)^{\frac{m_{1}}{2}+m_{2}}}{\left\|z_{1}\right\|^{m_{1}+m_{2}}} \\
& 1=\frac{\partial u_{\partial \Omega}}{\partial \eta^{+}}\left(z_{2}\right)-\frac{\partial u_{\partial \Omega}}{\partial \eta^{-}}\left(z_{2}\right) \geqslant \frac{\partial u_{\partial \Omega}}{\partial \eta^{+}}\left(z_{2}\right)=2 c_{n} \frac{\left(1-\left\|z_{2}\right\|\right)^{\frac{m_{1}}{2}+m_{2}}}{\left\|z_{2}\right\|^{m_{1}+m_{2}}} .
\end{aligned}
$$

It follows that $\frac{\left(1-\left\|z_{1}\right\|\right)^{\frac{m_{1}}{2}+m_{2}}}{\left\|z_{1}\right\|^{m_{1}+m_{2}}} \geqslant \frac{\left(1-\left\|z_{2}\right\|\right)^{\frac{m_{1}}{2}+m_{2}}}{\left\|z_{2}\right\|^{m_{1}+m_{2}}}$, that is $\left\|z_{1}\right\| \leqslant\left\|z_{2}\right\|$.

\section{Introduction}

\subsection{Motivation}

Le but de cette Note est d'établir des estimations du noyau de Green des boules hyperboliques réelles, complexes et quaternioniques sur des domaines relativement compacts a bord de classe $\mathrm{C}^{1+\alpha}$. Ces estimations nous permettent d'obtenir des informations sur la croissance des potentiels simple et double couche de ces domaines.

Ces résultats nous servent ensuite à démontrer que les boules géodésiques des boules hyperboliques réelles, complexes ou quaternioniques sont caractérisées par la propriété de la moyenne pour les fonctions harmoniques sur ces boules. De tels résultats ont déjà été obtenus dans le cas euclidien par H. Shahgholian [2] et dans le cas hyperbolique complexe par le premier auteur [1]. Nous verrons ici comment ces résultats s'étendent de façon unifiée aux cas hyperboliques réels et quaternioniques.

\subsection{Les boules hyperboliques}

Nous désignerons par $\mathbb{F}=\mathbb{R}, \mathbb{C}$ ou $\mathbb{H}$ le corps des réels, complexes ou quaternions, par $x \mapsto \bar{x}$ l'involution standard sur $\mathbb{F}$, par $|x|=\sqrt{x \bar{x}}$ la norme euclidienne sur $\mathbb{F}$ et enfin par $d=\operatorname{dim}_{\mathbb{R}} \mathbb{F}$. Soit un entier, $n \geqslant 3$ si $\mathbb{F}=\mathbb{R}$ et $n \geqslant 2$ si $\mathbb{F}=\mathbb{C}$ ou $\mathbb{H}$. Pour $x=\left(x_{1}, \ldots, x_{n+1}\right) \in \mathbb{F}^{n+1}$, définissons la forme quadratique $Q(x)=\left|x_{1}\right|^{2}+\ldots+\left|x_{n}\right|^{2}-\left|x_{n+1}\right|^{2}$. Alors, la composante connexe de l'identité $G$ du groupe des applications $\mathbb{F}$-linéaires qui préservent $Q$ et de déterminant 1 (sauf si $\mathbb{F}=\mathbb{H}$ ) est donnée par : si $\mathbb{F}=\mathbb{R}$, $G=S O_{0}(n, 1)$, si $\mathbb{F}=\mathbb{C}, G=S U(n, 1)$ et si $\mathbb{F}=\mathbb{H}, G=S p(n, 1)$. Soit $G=K A N$ la décomposition d'Iwasawa de $G$ et soit $M$ le centralisateur de $A$ dans $K$.

Nous considérons ici les espaces hyperboliques $G / K$, et plus précisément une réalisation bornée. Ainsi, pour $x=\left(x_{1}, \ldots, x_{n}\right)$ et $y=\left(y_{1}, \ldots, y_{n}\right)$ dans $\mathbb{F}^{n}$, posons $\langle x, y\rangle=x_{1} \overline{y_{1}}+\ldots+x_{n} \overline{y_{n}}$ et $\|x\|^{2}=$ $\langle x, x\rangle$. La boule unité $\mathbb{B}_{n}=\left\{x \in \mathbb{F}^{n}:\|x\|^{2}<1\right\}$ et son bord $\mathbb{S}^{n d-1}$ (la sphère unité dans $\mathbb{F}^{n}$ ) sont respectivement identifiés avec $G / K$ et $K / M$. Avec cette identification, $G$ agit transitivement sur $\mathbb{B}_{n}$ et sur $\mathbb{S}^{n d-1}$ par $g .\left(x_{1}, \ldots, x_{n}\right)=\left(y_{1} y_{n+1}^{-1}, \ldots, y_{n} y_{n+1}^{-1}\right)$ où $\left(y_{1}, \ldots, y_{n}, y_{n+1}\right)=g\left(x_{1}, \ldots, x_{n}, 1\right)$. Les boules $\mathbb{B}_{n}$ avec cette action de $G$ sont les espaces classiques de rang 1 de type non-compact (les boules hyperboliques réelles, complexes ou quaternioniques selon que $\mathbb{F}=\mathbb{R}, \mathbb{C}$ ou $\mathbb{H}$ ). Enfin, pour $z \in \mathbb{B}_{n}$, écrivons $P_{z}(\zeta)=\frac{\langle\zeta, z\rangle}{\langle z, z\rangle} z$ la projection sur $\mathbb{F} z$ et $Q_{z}=I-P_{z}$. Définissons

$$
\varphi_{z}(\zeta)=\frac{z-P_{z}(\zeta)-\sqrt{1-\|z\|^{2}} Q_{z}(\zeta)}{1-\langle\zeta, z\rangle}
$$

Alors $\varphi_{z}(\zeta)=g . \zeta$ pour le $g \in G$ tel que $g .0=z, g . z=0$. On vérifie aisément que

$$
1-\left\|\varphi_{z}(\zeta)\right\|^{2}=\frac{\left(1-\|z\|^{2}\right)\left(1-\|\zeta\|^{2}\right)}{|1-\langle\zeta, z\rangle|^{2}}, \quad \text { d'où } \quad\left\|\varphi_{z}(\zeta)\right\|^{2} \geqslant \frac{\left(1-\|z\|^{2}\right)\|z-\zeta\|^{2}}{|1-\langle\zeta, z\rangle|^{2}} \text {. }
$$




\section{Domenichino, P. Jaming}

Soit $\kappa$ la racine positive simple de $(G, A)$ et $m_{1}=d(n-1), m_{2}=d-1$ les multiplicités de $\kappa$ et $2 \kappa$ respectivement. Soit $\rho=\frac{m_{1}}{2}+m_{2}$, c'est-à-dire $\rho=\frac{n-1}{2}, n, 2 n+1$ selon que $\mathbb{F}=\mathbb{R}, \mathbb{C}$ ou $\mathbb{H}$. La mesure de Lebesgue sur $\mathbb{B}_{n}$ sera notée $d x$ et la mesure $G$-invariante sur $\mathbb{B}_{n}$ est alors $d \mu(x)=\frac{d x}{\left(1-|x|^{2}\right)^{\rho}}$.

Soit $D_{F}$ l'opérateur de Laplace-Beltrami sur $\mathbb{B}_{n}$. Une fonction $f$ sur $\mathbb{B}_{n}$ telle que $D_{\mathbb{F}} f=0$ est alors appelée $D_{\mathbb{F}}$-harmonique. La fonction de Green $\Gamma$ pour $D_{\mathbb{F}}$, c'est-à-dire la fonction $\mathbb{B}_{n} \times \mathbb{B}_{n} \rightarrow \mathbb{F}$ qui vérifie l'équation (en $z) D_{\mathbb{F}} \Gamma(\zeta, z)=0$ sur $\mathbb{B}_{n} \backslash\{\zeta\}$, est donnée $\operatorname{par} \Gamma(\zeta, z)=-c_{n} \int_{\left\|\varphi_{z}(\zeta)\right\|^{2}}^{1} \frac{(1-t)^{\frac{m_{1}}{2}+m_{2}-1}}{t^{1+m_{1}+m_{2}}} d t$ avec $c_{n}$ une constante positive, qu'on notera $\Gamma(\zeta, z)=-c_{n} \gamma\left(\left\|\varphi_{z}(\zeta)\right\|^{2}\right)$

\section{Potentiels simple et double couche}

\subsection{Description des domaines}

Dans cette note, $\Omega$ sera un ouvert relativement compact dans $\mathbb{B}_{n}$ de bord $\partial \Omega$ de classe $\mathcal{C}^{1+\alpha}(\alpha>0)$ et tel que $\partial \Omega=\partial \bar{\Omega}$. Nous noterons $\Omega^{-}=\Omega$ et $\Omega^{+}=\mathbb{B}_{n} \backslash \bar{\Omega}$. Alors $\Omega$ est donné par une fonction définissante $\rho$ de classe $\mathcal{C}^{1+\alpha}$ sur $\mathbb{B}_{n}$ telle que $\Omega^{-}=\left\{z \in \mathbb{B}_{n}: \rho(z)<0\right\}, \partial \Omega=\left\{z \in \mathbb{B}_{n}: \rho(z)=0\right\}$ et $\Omega^{+}=\left\{z \in \mathbb{B}_{n}: \rho(z)>0\right\}$. Pour $x \in \partial \Omega$, notons $\eta_{x}^{g}$ la normale extérieure à $\partial \Omega$ en $x$ pour la métrique hyperbolique. Comme $\partial \Omega$ est une hypersurface compacte de classe $\mathcal{C}^{1+\alpha}$, il existe un voisinage $U$ de $\partial \Omega$ et un $\varepsilon>0$ telle que $F: \begin{array}{ccc}\partial \Omega \times(-\varepsilon, \varepsilon) & \rightarrow U \\ (x, t) & \mapsto x+t \eta_{x}^{g}\end{array}$ est un homéomorphisme de classe $\mathrm{C}^{\alpha}$. En particulier, chaque $y \in U$ appartient à une unique normale à $\partial \Omega$ et nous appellerons $y_{0} \in \partial \Omega$ la base de cette normale i.e. $y=y_{0}+t \eta_{y_{0}}^{g}$. Il existe alors une constante $C>0$ telle que pour tout $y_{0} \in \partial \Omega$ et pour tout $y \in U$, tels que $y=y_{0}+t \eta_{y_{0}}^{g},\|y-\zeta\| \geqslant C\left\|y_{0}-\zeta\right\|$, pour tout $\zeta \in \partial \Omega$. Enfin, notons $d \sigma_{g}$ la contraction de $d \mu$ par rapport à la normale extérieure à $\partial \Omega$ pour la métrique hyperbolique.

\subsection{Potentiel simple couche.}

Définissons le $D_{\mathbb{F}}$-potentiel simple couche de $\partial \Omega$ par $u_{\partial \Omega}(z)=\int_{\partial \Omega} \Gamma(\zeta, z) d \sigma_{g}(\zeta)$. Comme dans [1], on montre alors :

Proposition 2.1. - Le potentiel $u_{\partial \Omega}$ est bien défini et continu sur $\mathbb{B}_{n}$.

Preuve. La preuve est la même que dans [1], pour peu qu'on remplace l'estimation du noyau de Green complexe (13) par l'estimation plus générale vérifiée sur tout compact $K$ de $\mathbb{B}_{n}$ :

$$
\|\Gamma(\zeta, z)\| \leqslant C_{K} \frac{|1-\langle\zeta, z\rangle|^{m_{1}+m_{2}-1}}{\left(1-\|z\|^{2}\right)^{\frac{m_{1}+m_{2}-1}{2}}\|z-\zeta\|^{m_{1}+m_{2}-1}} \leqslant C_{K} \frac{1}{\|z-\zeta\|^{m_{1}+m_{2}-1}},
$$

pour $z \in K, \zeta \in \partial \Omega$.

\subsection{Saut de la dérivée normale}

La proposition centrale de cette note est alors la suivante :

Proposition 2.2. - Pour tout compact $K$ de $\mathbb{B}_{n}$, il existe alors une constante $C_{K}$ telle que, pour tout $z \in K, \zeta \in \partial \Omega$ et pour tout $i=1, \ldots, n$ on a

et

$$
\left|\frac{\partial \Gamma}{\partial z_{i}}(z, \zeta)+\frac{\partial \Gamma}{\partial \zeta_{i}}(z, \zeta)\right| \leqslant \frac{C_{K}}{\|z-\zeta\|^{m_{1}+m_{2}-1}}=\frac{C_{K}}{\|z-\zeta\|^{n d-2}}
$$

$$
\left|\frac{\partial \Gamma}{\partial z_{i}}(z, \zeta)\right| \leqslant \frac{C_{K}}{\|z-\zeta\|^{m_{1}+m_{2}}}=\frac{C_{K}}{\|z-\zeta\|^{n d-1}} .
$$


Preuve. Il suffit de voir que

$$
\begin{aligned}
\frac{\partial \Gamma}{\partial z_{i}}(z, \zeta) & =c_{n} \frac{\left(1-\left\|\varphi_{z}(\zeta)\right\|^{2}\right)^{\frac{m_{1}}{2}+m_{2}-1}}{\left\|\varphi_{z}(\zeta)\right\|^{m_{1}+m_{2}+1}} \frac{\partial\left\|\varphi_{z}\right\|^{2}}{\partial z_{i}}(\zeta) \\
& =c_{n} \frac{\left(1-\left\|\varphi_{z}(\zeta)\right\|^{2}\right)^{\frac{m_{1}}{2}+m_{2}-1}}{\left\|\varphi_{z}(\zeta)\right\|^{m_{1}+m_{2}+1}|1-\langle\zeta, z\rangle|^{2}} \times\left(1-\|\zeta\|^{2}\right) \frac{\overline{z_{i}}(1-\langle\zeta, z\rangle)-\overline{\zeta_{i}}\left(1-\|z\|^{2}\right)}{1-\langle\zeta, z\rangle}
\end{aligned}
$$

Comme $(z, \zeta) \in K \times \partial \Omega$ un compact dans $\mathbb{B}_{n} \times \mathbb{B}_{n}$, et comme $|1-\langle\zeta, z\rangle|$ ne s'annule que pour $z=\zeta \in$ $\mathbb{S}^{n d-1}$, on a la minoration $|1-\langle\zeta, z\rangle| \geqslant C$. Avec (1.2), il vient alors

$$
c_{n} \frac{\left(1-\left\|\varphi_{z}(\zeta)\right\|^{2}\right)^{\frac{m_{1}}{2}+m_{2}-1}}{\left\|\varphi_{z}(\zeta)\right\|^{m_{1}+m_{2}+1}|1-\langle\zeta, z\rangle|^{2}} \leqslant C_{K} \frac{1}{\|z-\zeta\|^{m_{1}+m_{2}+1}} .
$$

D'après (2.5) et (2.6), pour démontrer (2.3), il nous reste à estimer

$$
\left(1-\|\zeta\|^{2}\right) \frac{\overline{z_{i}}(1-\langle\zeta, z\rangle)-\overline{\zeta_{i}}\left(1-\|z\|^{2}\right)}{1-\langle\zeta, z\rangle}+\left(1-\|z\|^{2}\right) \frac{\overline{\zeta_{i}}(1-\langle z, \zeta\rangle)-\overline{z_{i}}\left(1-\|\zeta\|^{2}\right)}{1-\langle z, \zeta\rangle}
$$

qu'on réécrit $\overline{z_{i}}\left(1-\|\zeta\|^{2}\right) \frac{\|z\|^{2}-\langle\zeta, z\rangle}{1-\langle\zeta, z\rangle}+\overline{\zeta_{i}}\left(1-\|z\|^{2}\right) \frac{\|\zeta\|^{2}-\langle z, \zeta\rangle}{1-\langle z, \zeta\rangle}$ puis

$$
\left(\overline{z_{i}}-\bar{\zeta}_{i}\right)\left(1-\|\zeta\|^{2}\right) \frac{\langle z-\zeta, z\rangle}{1-\langle\zeta, z\rangle}+\overline{\zeta_{i}}\left[\left(1-\|z\|^{2}\right) \frac{\langle\zeta-z, \zeta\rangle}{1-\langle z, \zeta\rangle}+\left(1-\|\zeta\|^{2}\right) \frac{\langle z-\zeta, z\rangle}{1-\langle\zeta, z\rangle}\right] .
$$

Le premier terme est évidemment en $O\left(\|z-\zeta\|^{2}\right)$, alors que le second terme s'écrit $\overline{\zeta_{i}}\langle\zeta-z, \xi\rangle$ avec

$$
\xi=(\zeta-z) \frac{1-\|z\|^{2}}{1-\langle z, \zeta\rangle}+z \frac{\left(\|\zeta\|^{2}-\|z\|^{2}\right)\left(1+\|\zeta\|^{2}+\|z\|^{2}\right)+\langle\zeta-z, z\rangle\|z\|^{2}-\langle z-\zeta, \zeta\rangle\|\zeta\|^{2}}{|1-\langle z, \zeta\rangle|^{2}} .
$$

Par suite $\|\xi\|=O(\|z-\zeta\|)$ et le second terme de (2.7) est aussi un $O\left(\|z-\zeta\|^{2}\right)$.

Pour l'inégalité (2.4), il suffit de voir que $\overline{z_{i}}(1-\langle z, \zeta\rangle)-\overline{\zeta_{i}}(1-\langle\zeta, z\rangle)=\left(\overline{z_{i}}-\overline{\zeta_{i}}\right)\langle z, z-\zeta\rangle$, qui est donc un $O(\|z-\zeta\|)$, puis d'utiliser l'inégalité (2.6).

Définissons le $D_{\mathbb{F}}$-potentiel double couche de $\partial \Omega \operatorname{par} v_{\partial \Omega}(z)=\int_{\partial \Omega}\left\langle\operatorname{grad}_{\zeta} \Gamma(\zeta, z), \eta_{\zeta}\right\rangle d \sigma_{g}(\zeta)$. En reprenant la preuve de la proposition 3 de [1], basée sur la formule de Green pour $D_{\mathbb{F}}$, on voit aisément que

$$
v_{\partial \Omega}(z)=0, \text { pour } z \in \Omega^{+} \text {et } v_{\partial \Omega}(z)=1, \text { pour } z \in \Omega^{-} .
$$

Nous allons maintenant nous intéresser aux limites

$$
\begin{aligned}
\frac{\partial u_{\partial \Omega}}{\partial \eta^{+}}\left(z_{0}\right)= & \lim _{\substack{z \rightarrow z_{0}, z \in \Omega^{+} \\
z=z_{0}+t \eta_{z_{0}}}} \int_{\partial \Omega}\left\langle\operatorname{grad}_{z} \Gamma(\zeta, z), \eta_{z_{0}}\right\rangle d \sigma_{g}(\zeta)=\lim _{\substack{z \rightarrow z_{0}, z \in \Omega^{+} \\
z=z_{0}+t \eta_{z_{0}}}} v_{\partial \Omega}(z) \\
\frac{\partial u_{\partial \Omega}}{\partial \eta^{-}}\left(z_{0}\right)= & \lim _{\substack{z \rightarrow z_{0}, z \in \Omega^{-} \\
z=z_{0}+t \eta_{z_{0}}}} \int_{\partial \Omega}\left\langle\operatorname{grad}_{z} \Gamma(\zeta, z), \eta_{z_{0}}\right\rangle d \sigma_{g}(\zeta)=\lim _{\substack{z \rightarrow z_{0}, z \in \Omega^{-} \\
z=z_{0}+t \eta_{z_{0}}}} v_{\partial \Omega}(z) .
\end{aligned}
$$

Fixons $\varepsilon>0$. Soit $z_{0} \in \partial \Omega$, pour $z \in \Omega^{+} \cap U, z=z_{0}+t \eta_{z_{0}}, t>0$ et pour $z^{\prime} \in \Omega^{-} \cap U, z^{\prime}=z_{0}+\tau \eta_{z_{0}}$, $\tau<0$, considérons $v_{\partial \Omega}(z)-v_{\partial \Omega}\left(z^{\prime}\right)=\int_{\partial \Omega}\left(\left\langle\operatorname{grad}_{z} \Gamma(\zeta, z), \eta_{z_{0}}\right\rangle-\left\langle\operatorname{grad}_{z^{\prime}} \Gamma\left(z^{\prime}, \zeta\right), \eta_{z_{0}}\right\rangle\right) d \sigma_{g}(\zeta)$. 


\section{Domenichino, P. Jaming}

Pour $0<\delta<\frac{1}{2} d\left(\zeta, \mathbb{S}^{n d-1}\right)$, notons $S_{\delta}=\left\{\zeta \in \partial \Omega:\left\|\zeta-z_{0}\right\| \leqslant \delta\right\}$, qui est alors compact dans $\mathbb{B}_{n}$. Réécrivons la dernière intégrale à l'aide de (2.8) :

$$
\begin{gathered}
\int_{S_{\delta}}\left\langle\operatorname{grad}_{z} \Gamma(\zeta, z), \eta_{z_{0}}-\eta_{\zeta}\right\rangle d \sigma_{g}(\zeta)-\int_{S_{\delta}}\left\langle\operatorname{grad}_{z^{\prime}} \Gamma\left(z^{\prime}, \zeta\right), \eta_{z_{0}}-\eta_{\zeta}\right\rangle d \sigma_{g}(\zeta)+ \\
+\int_{S_{\delta}}\left[\left\langle\operatorname{grad}_{z} \Gamma(\zeta, z)+\operatorname{grad}_{\zeta} \Gamma(\zeta, z), \eta_{\zeta}\right\rangle-\left\langle\operatorname{grad}_{z^{\prime}} \Gamma\left(z^{\prime}, \zeta\right)+\operatorname{grad}_{\zeta} \Gamma\left(z^{\prime}, \zeta\right), \eta_{\zeta}\right\rangle\right] d \sigma_{g}(\zeta)+ \\
+\int_{\partial \Omega \backslash S_{\delta}}\left\langle\operatorname{grad}_{z} \Gamma(\zeta, z)+\operatorname{grad}_{\zeta} \Gamma(\zeta, z), \eta_{\zeta}\right\rangle-\left\langle\operatorname{grad}_{z^{\prime}} \Gamma\left(z^{\prime}, \zeta\right)+\operatorname{grad}_{\zeta} \Gamma\left(z^{\prime}, \zeta\right), \eta_{\zeta}\right\rangle d \sigma_{g}(\zeta)+ \\
+\int_{\partial \Omega \backslash S_{\delta}}\left(\left\langle\operatorname{grad}_{z} \Gamma(\zeta, z), \eta_{z_{0}}-\eta_{\zeta}\right\rangle-\left\langle\operatorname{grad}_{z^{\prime}} \Gamma\left(\zeta, z^{\prime}\right), \eta_{z_{0}}-\eta_{\zeta}\right\rangle\right) d \sigma_{g}(\zeta)+1
\end{gathered}
$$

Ensuite, en procédant comme dans [1], mais en utilisant les estimations (2.3)-(2.4), on voit que, pour $z \in$ $\left\{\omega \in \mathbb{B}_{n}: \omega=z_{0}+t \eta_{z_{0}}, t \in[-\varepsilon, \varepsilon],\left\|\omega-z_{0}\right\| \leqslant \delta\right\}$ et $\zeta \in \partial \Omega$, le terme

$$
\left\langle\operatorname{grad}_{z} \Gamma(\zeta, z)+\operatorname{grad}_{\zeta} \Gamma(\zeta, z), \eta_{\zeta}\right\rangle
$$

est uniformément dominé par $\frac{1}{\|z-\zeta\|^{n d-2}}$. Par conséquent, c'est un $O\left(\frac{1}{\left\|z_{0}-\zeta\right\|^{n d-2}}\right)$ et l'intégrale (2.10) est en $\mathrm{O}(\delta)$. Ensuite, en utilisant (2.4) et la régularité de $\partial \Omega$, on $\operatorname{montre}$ que $\left\langle\operatorname{grad}_{z} \Gamma(\zeta, z), \eta_{z_{0}}-\eta_{\zeta}\right\rangle$ est un $O\left(\frac{1}{\left\|z_{0}-\zeta\right\|^{n d-1-\alpha}}\right)$. Ainsi, les intégrales (2.9) sont en $\mathrm{O}\left(\delta^{\alpha}\right)$. En particulier, pour un bon choix de $\delta$, les intégrales (2.9)-(2.10) sont $\leqslant \varepsilon$.

Enfin, les intégrales (2.11)-(2.12) sont continues en $z, z^{\prime}$ pour peu que $\left\|z-z_{0}\right\|<\frac{\delta}{2},\left\|z^{\prime}-z_{0}\right\|<\frac{\delta}{2}$ on peut donc également les rendre $<\varepsilon$. Nous avons ainsi démontré

Proposition 2.3. - Avec les notations ci-dessus, pour tout $z_{0} \in \partial \Omega, \frac{\partial u_{\partial \Omega}}{\partial \eta^{+}}\left(z_{0}\right)-\frac{\partial u_{\partial \Omega}}{\partial \eta^{-}}\left(z_{0}\right)=1$.

\section{Caractérisation des boules géodésiques par la propriété de la moyenne}

Les estimations de la section précédente nous permettent de démontrer le théorème suivant :

THÉORÈME 3.1. - Soit $\Omega \subset \mathbb{B}_{n}$ un domaine relativement compact à bord $\partial \Omega$ de classe $\mathrm{e}^{1+\alpha}, \alpha>0$ et tel que $\partial \Omega=\partial \bar{\Omega}$ et soit $a \in \Omega$. Supposons que pour toute fonction $f D_{\mathbb{F}}$-harmonique au voisinage de $\bar{\Omega}$ on ait $f(a)=\frac{1}{\sigma_{g}(\partial \Omega)} \int_{\partial \Omega} f(\zeta) d \sigma_{g}(\zeta)$ alors $\Omega$ est une boule hyperbolique centrée en $a$, c'est-à-dire $\Omega=\varphi_{a}\left(B_{r}\right)$ où $B_{r}$ est une boule euclidienne centrée en 0 .

Esquisse de preuve. La preuve suit de près celle de [1] et est inspirée de celle de [2]. Nous avons inclus les détails dans la version anglaise.

Second author partially supported by the European Commission (TMR 1998-2001 Network Harmonic Analysis).

\section{Références bibliographiques}

[1] Domenichino C., Converse of spherical mean-value property for invariant harmonic functions, Complex Variables (2001) (à paraître).

[2] Shahgholian H., A characterization of the sphere in terms of single-layer potentials, Proc. Amer. Math. Soc. 115 (4) 1167-1168. 